\title{
CARTAN-EILENBERG GORENSTEIN FLAT COMPLEXES
}

\author{
GANG YANG and LI LIANG ${ }^{* \dagger}$
}

\begin{abstract}
In this paper, we study Cartan-Eilenberg Gorenstein flat complexes. We show that over coherent rings a Cartan-Eilenberg Gorenstein flat complex can be gotten by a so-called complete CartanEilenberg flat resolution. We argue that over a coherent ring every complex has a Cartan-Eilenberg Gorenstein flat cover.
\end{abstract}

\section{Introduction and Preliminaries}

In his thesis Verdier introduced the notion of a Cartan-Eilenberg injective complex (Definition 4.6.1 of [17]) and considered the so called Cartan-Eilenberg injective and projective resolutions of complexes. In [4], using the ideas of Verdier, Enochs further showed that Cartan-Eilenberg resolutions can be defined in terms of preenvelopes and precovers by Cartan-Eilenberg injective and projective complexes. Also, Enochs considered Cartan-Eilenberg flat complexes which are obvious extension of Cartan-Eilenberg projective complexes and showed that they are precisely the direct limits of the finitely generated Cartan-Eilenberg projective complexes. In this paper, we continue to study Cartan-Eilenberg flat complexes and then Cartan-Eilenberg Gorenstein flat complexes. We describe how the homological theory on Gorenstein flat modules generalizes to a homological theory on Cartan-Eilenberg Gorenstein flat complexes.

Throughout, let $R$ be an associative ring with 1, $R$-Mod (respectively, Mod$R$ ) the category of left (respectively, right) $R$-modules and $C(R$-Mod) (respectively, $C$ (Mod- $R$ )) the category of complexes of left (respectively, right) $R$-modules. Unless stated otherwise, an $R$-module (respectively, $R$-complex) will be understood to be a left $R$-module (respectively, a complex of left $R$ modules).

\footnotetext{
* This work was supported by the Science and Technology Program of Gansu Province of China (Grant No. 1107RJZA233) and NSF of China (Grant No. 11101197; 11201376; 11301240).

${ }^{\dagger}$ The authors thank the referee for his/her careful reading and many constructive suggestions, which have improved the present article.

Received 14 January 2012, in final form 15 August 2012.
} 
To every complex $C=\cdots \rightarrow C_{m+1} \stackrel{\delta_{m+1}^{c}}{\longrightarrow} C_{m} \stackrel{\delta_{m}^{c}}{\longrightarrow} C_{m-1} \rightarrow \cdots$, the $m$ th cycle of $C$ is defined as $\operatorname{Ker}\left(\delta_{m}^{C}\right)$ and is denoted by $\mathrm{Z}_{m}(C)$, the $m$ th boundary is defined as $\operatorname{Im}\left(\delta_{m+1}^{C}\right)$ and is denoted by $\mathrm{B}_{m}(C)$. We use $\mathrm{Z}(C), \mathrm{B}(C) \subseteq C$ to denote the subcomplexes of cycles and boundaries of the complex $C$, and $\mathrm{H}(C)=\mathrm{Z}(C) / \mathrm{B}(C)$ to denote the homology complex of $C$. For a complex $C$, the suspension of $C$, denoted by $\Sigma C$, is the complex given by $(\Sigma C)_{m}=C_{m-1}$ and $\delta_{m}^{\Sigma C}=-\delta_{m-1}^{C}$. The complex $\Sigma(\Sigma C)$ is denoted by $\Sigma^{2} C$ and inductively we define $\Sigma^{m} C$ for all $m \in Z$ Z. In the paper, we use subscripts to distinguish complexes. For example, if $C_{\alpha}$ is a complex with the subscript $\alpha$, then $C_{\alpha}$ will be

$$
\cdots \rightarrow\left(C_{\alpha}\right)_{m+1} \stackrel{\delta_{m+1}}{\longrightarrow}\left(C_{\alpha}\right)_{m} \stackrel{\delta_{m}}{\longrightarrow}\left(C_{\alpha}\right)_{m-1} \stackrel{\delta_{m-1}}{\longrightarrow}\left(C_{\alpha}\right)_{m-2} \rightarrow \cdots .
$$

If $M$ is an $R$-module then $M$ can be regarded as a complex concentrated at 0 . We will denote this complex by $\underline{M}$. So $\underline{M}=\cdots \rightarrow 0 \rightarrow M \rightarrow 0 \rightarrow \cdots$ with $M$ in the 0th degree. Similarly we denote the complex $\bar{M}=\cdots \rightarrow 0 \rightarrow$ $M \rightarrow M \rightarrow 0 \rightarrow \cdots$ with $M$ in the 1 and 0th degrees.

Given two complexes $X$ and $Y$, we let $\mathscr{C} \mathrm{om}(X, Y)$ denote the complex of Z-modules

$$
\cdots \rightarrow \prod_{i \in \mathrm{Z}} \operatorname{Hom}_{R}\left(X_{i}, Y_{i+n}\right) \stackrel{\delta_{n}}{\longrightarrow} \prod_{i \in \mathrm{Z}} \operatorname{Hom}_{R}\left(X_{i}, Y_{i+n-1}\right) \rightarrow \cdots,
$$

where $\delta_{n}\left(\left(f_{i}\right)_{i \in \mathrm{Z}}\right)=\left(\delta_{i+n}^{Y} f_{i}-(-1)^{n} f_{i-1} \delta_{i}^{X}\right)_{i \in \mathrm{Z}}$. We say $f: X \rightarrow Y$ a morphism of complexes if $f=\left(f_{i}\right)_{i \in Z} \in \prod_{i \in Z} \operatorname{Hom}_{R}\left(X_{i}, Y_{i}\right)$ and $\delta_{i}^{Y} f_{i}=f_{i-1} \delta_{i}^{X}$ for all $i \in \mathrm{Z}$. The set of all morphisms from $X$ to $Y$ is denoted by $\operatorname{Hom}(X, Y)$. Let $\underline{\operatorname{Hom}}(X, Y)=\mathrm{Z}(\mathscr{H} \mathrm{om}(X, Y))$, that is, $\underline{\operatorname{Hom}}(X, Y)$ is the complex of Zmodules with $n$th component $\operatorname{Hom}(X, Y)_{n}=Z_{n}(\mathscr{H}$ om $(X, Y))=\operatorname{Hom}(X$, $\left.\Sigma^{-n} Y\right)$ and differential $\lambda_{n}: \underline{\operatorname{Hom}}(X, Y)_{n} \rightarrow \underline{\operatorname{Hom}}(X, Y)_{n-1}$ is defined by

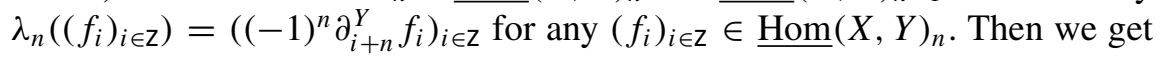
new functors $\underline{\operatorname{Hom}}(X,-)$ and $\underline{\operatorname{Hom}}(-, Y)$ which are left exact and have right derived functors whose values will be complexes. These functors should certainly be denoted by $\underline{\operatorname{Ext}}^{i}(-,-)$. It is easy to see that $\underline{\operatorname{Ext}}^{i}(X, Y)$ is the complex

$$
\cdots \rightarrow \operatorname{Ext}^{i}\left(X, \Sigma^{n-1} Y\right) \rightarrow \operatorname{Ext}^{i}\left(X, \Sigma^{n} Y\right) \rightarrow \operatorname{Ext}^{i}\left(X, \Sigma^{n+1} Y\right) \rightarrow \cdots
$$

with differential induced by the differential of $Y$.

If $X$ is a complex of right $R$-modules and $Y$ is a complex of left $R$-modules, then their tensor product $X \otimes Y$ is defined by $(X \otimes Y)_{n}=\bigoplus_{i+j=n} X_{i} \otimes_{R} Y_{j}$ in degree $n$, the differential $\delta_{n}$ is defined by $\delta^{X}(x) \otimes y+(-1)^{|x|} x \otimes \delta^{Y}(y)$ on the generators, where $|x|$ is the degree of the element $x$. Let $X \otimes Y=$ $\frac{(X \otimes Y)}{\mathrm{B}(X \otimes Y)}$, that is, $X \otimes Y$ is the complex of Z-modules with $n$th component 
$(X \otimes Y)_{n}=\frac{(X \otimes Y)_{n}}{\mathrm{~B}_{n}(X \otimes Y)}$ and differential $\lambda_{n}:(X \otimes Y)_{n} \rightarrow(X \otimes Y)_{n-1}$ given by $\lambda_{n}(\overline{x \otimes y})=\overline{\delta^{X}(x) \otimes y}$, where $\overline{x \otimes y}$ is used to denote the coset in $\frac{(X \otimes Y)_{n}}{\mathrm{~B}_{n}(X \otimes Y Y)}$. Since the category of complexes have enough projectives, and $-\otimes Y$ and $X \otimes-$ are right exact, we can construct left derived functors which we denote by $\operatorname{Tor}_{i}(-,-)$.

The next result can be found in [6, Proposition 2.1].

Lemma 1.1. Let $Y, Z$ be two complexes and $X$ a complex of right $R$-modules. Then we have the following natural isomorphisms.

(1) $\underline{\operatorname{Hom}}(X \otimes Y, Z) \cong \underline{\operatorname{Hom}}(X, \underline{\operatorname{Hom}}(Y, Z))$.

(2) $\left(\lim _{i} X_{i}\right) \otimes Y \cong \lim \left(X_{i} \otimes Y\right)$ for a direct family $\left\{X_{i}\right\}$ of complexes of right $R$-modules.

(3) For an R-module $M, \underline{\operatorname{Hom}}\left(\Sigma^{m} \bar{M}, Y\right) \cong \Sigma^{-1-m} \operatorname{Hom}_{R}(M, Y)$ and $\underline{\operatorname{Hom}}\left(Y, \Sigma^{m} \bar{M}\right) \cong \Sigma^{-m} \operatorname{Hom}_{R}(Y, M)$.

In the sequel we give some other definitions for use later.

Definition 1.2. An $R$-module $M$ is called Gorenstein injective if there exists an exact sequence

$$
\cdots \rightarrow I_{2} \rightarrow I_{1} \rightarrow I_{0} \rightarrow I_{-1} \rightarrow I_{-2} \rightarrow \cdots
$$

of injective $R$-modules with $M=\operatorname{Ker}\left(I_{-1} \rightarrow I_{-2}\right)$, such that it remains exact after applying $\operatorname{Hom}_{R}(I,-)$ for any injective $R$-module $I$.

Definition 1.3. An $R$-module $N$ is called Gorenstein flat if there exists an exact sequence

$$
\cdots \rightarrow F_{2} \rightarrow F_{1} \rightarrow F_{0} \rightarrow F_{-1} \rightarrow F_{-2} \rightarrow \cdots
$$

of flat $R$-modules with $N=\operatorname{Ker}\left(F_{-1} \rightarrow F_{-2}\right)$, such that it remains exact after applying $I \otimes_{R}$ - for any injective right $R$-module $I$.

The Gorenstein flat modules were introduced by Enochs, Jenda and Torrecillas in 1990's [9] as generalizations of the classical flat modules. Over Gorenstein rings, such modules were shown to have many properties similar to those of the classical flat modules over general rings. Lately, Gorenstein flat modules over more general rings have been studied by many authors such as Ding and Chen [3], Holm [13], Bennis [2], and Yang and Liu [18] etc.

The following two definitions come from [4].

Definition 1.4. Given a class $\mathscr{F}$ of $R$-modules. A complex $A$ is called a Cartan-Eilenberg (C-E for short) $\mathscr{F}$ complex if $A, \mathrm{Z}(A), \mathrm{B}(A)$ and $\mathrm{H}(A)$ are all in $C(\mathscr{F})$, where $C(\mathscr{F})$ denotes the class of complexes with each component in 
$\mathscr{F}$. In particular, if the class $\mathscr{F}$ consists of all injective $R$-modules then a C-E $\mathscr{F}$ complex is just called a $\mathrm{C}$-E injective complex. Also, we use the obvious modifications, e.g. C-E projective, C-E flat, C-E Gorenstein injective and C-E Gorenstein flat complexes, of such names. We let $\mathrm{CE}(\mathscr{F})$ denote the class of $\mathrm{C}$-E $\mathscr{F}$ complexes for a given class $\mathscr{F}$ of $R$-modules.

Definition 1.5. A sequence of complexes $\cdots \rightarrow C_{1} \rightarrow C_{0} \rightarrow C_{-1} \rightarrow$ $\cdots$ is said to be $\mathrm{C}-\mathrm{E}$ exact if

(1) $\cdots \rightarrow C_{1} \rightarrow C_{0} \rightarrow C_{-1} \rightarrow \cdots$,

(2) $\cdots \rightarrow \mathrm{Z}\left(C_{1}\right) \rightarrow \mathrm{Z}\left(C_{0}\right) \rightarrow \mathrm{Z}\left(C_{-1}\right) \rightarrow \cdots$,

(3) $\cdots \rightarrow \mathrm{B}\left(C_{1}\right) \rightarrow \mathrm{B}\left(C_{0}\right) \rightarrow \mathrm{B}\left(C_{-1}\right) \rightarrow \cdots$,

(4) $\cdots \rightarrow C_{1} / \mathrm{Z}\left(C_{1}\right) \rightarrow C_{0} / \mathrm{Z}\left(C_{0}\right) \rightarrow C_{-1} / \mathrm{Z}\left(C_{-1}\right) \rightarrow \cdots$,

(5) $\cdots \rightarrow C_{1} / \mathrm{B}\left(C_{1}\right) \rightarrow C_{0} / \mathrm{B}\left(C_{0}\right) \rightarrow C_{-1} / \mathrm{B}\left(C_{-1}\right) \rightarrow \cdots$,

(6) $\cdots \rightarrow \mathrm{B}\left(C_{1}\right) \rightarrow \mathrm{H}\left(C_{0}\right) \rightarrow \mathrm{H}\left(C_{-1}\right) \rightarrow \cdots$

are all exact.

REMARK 1.6. In the above definition, exactness of (1) and (2) implies exactness of all (1)-(6), and exactness of (1) and (5) implies exactness of all (1)-(6).

Given two complexes $X$ and $Y$. It follows from [4, Theorems 5.5 and 5.7] that there exist two $\mathrm{C}$-E exact sequences $\cdots \rightarrow P_{2} \rightarrow P_{1} \rightarrow P_{0} \rightarrow X \rightarrow 0$ and $0 \rightarrow Y \rightarrow I^{0} \rightarrow I^{1} \rightarrow I^{2} \rightarrow \cdots$, where each $P_{n}$ is a C-E projective complex and each $I^{n}$ is a C-E injective complex. By [4, Proposition 6.3], we can compute derived functors of $\operatorname{Hom}(-,-)$ using either of the two sequences. We denote these derived functors as $\overline{\operatorname{Ext}}^{n}(X, Y)$. Now one can easily check that for any $\mathrm{C}$-E exact sequence $0 \rightarrow A \rightarrow B \rightarrow C \rightarrow 0$, there exist exact sequences

$$
0 \rightarrow \operatorname{Hom}(X, A) \rightarrow \operatorname{Hom}(X, B) \rightarrow \operatorname{Hom}(X, C) \rightarrow \overline{\operatorname{Ext}}^{1}(X, A) \rightarrow \cdots
$$

and

$$
0 \rightarrow \operatorname{Hom}(C, Y) \rightarrow \operatorname{Hom}(B, Y) \rightarrow \operatorname{Hom}(A, Y) \rightarrow \overline{\operatorname{Ext}}^{1}(C, Y) \rightarrow \cdots
$$

\section{C-E flat complexes}

In this section we give some characterizations of $\mathrm{C}$-E flat complexes that will be used in Section 3. We prove that $R$ is right coherent if and only if every complex of $R$-modules has a C-E flat preenvelope.

We recall from [6] that a complex $F$ is flat if the functor $-\otimes F$ is exact. Equivalently, a complex $F$ is flat if and only if $\operatorname{Tor}_{1}(X, F)=0$ for any complex 
$X$ of right $R$-modules if and only if it is exact and for each $i \in \mathrm{Z}, \mathrm{Z}_{i} F$ is a flat $R$-module.

Lemma 2.1. Let $P$ be a C-E projective complex. Then $-\otimes P$ is exact for any short $\mathrm{C}-\mathrm{E}$ exact sequence.

Proof. By [4, Proposition 3.4], we note that every C-E projective complex can be written as $\left(\oplus_{i \in \mathrm{Z}} \Sigma^{i} \overline{K_{i}}\right) \bigoplus\left(\oplus_{i \in \mathrm{Z}} \Sigma^{i} L_{i}\right)$, where $K_{i}$ and $L_{i}$ are projective $R$-modules. Thus we need only to show that $-\otimes \Sigma^{i} Q$ and $-\otimes \Sigma^{i} \bar{Q}$ are exact for any $\mathrm{C}$-E exact sequence, where $Q$ is a projective $R$-module.

Let $0 \rightarrow A \rightarrow B \rightarrow C \rightarrow 0$ be a short C-E exact sequence of complexes of right $R$-modules. Since $\Sigma^{i} \bar{Q}$ is a flat complex, we get that $-\otimes \Sigma^{i} \bar{Q}$ is exact for any exact sequence of complexes. Note that $Q$ is a projective $R$-module, then one can check easily that the sequence $0 \rightarrow A \otimes \underline{Q} \rightarrow B \otimes \underline{Q} \rightarrow C \otimes \underline{Q} \rightarrow 0$ is $\mathrm{C}-\mathrm{E}$ exact, and so we have the exact sequence

$$
\begin{aligned}
0 \rightarrow(A \otimes \underline{Q}) / B(A \otimes \underline{Q}) \rightarrow(B \otimes \underline{Q}) / B(B \otimes \underline{Q}) & \\
\rightarrow & (C \otimes \underline{Q}) / B(C \otimes \underline{Q}) \rightarrow 0 .
\end{aligned}
$$

This shows that the sequence $0 \rightarrow A \otimes Q \rightarrow B \otimes Q \rightarrow C \otimes Q \rightarrow 0$ is exact, and hence the sequence $0 \rightarrow A \otimes \Sigma^{i} \underline{Q} \rightarrow B \otimes \overline{\Sigma^{i}} \underline{Q} \rightarrow C \bar{\otimes} \Sigma^{i} \underline{Q} \rightarrow 0$ is exact. Thus the functor $-\otimes \Sigma^{i} \underline{Q}$ is exact for any $\mathrm{C}$-E exact sequence.

Given a complex $C$, we let $C^{+}$stand for the character complex $\underline{\operatorname{Hom}}(C, \overline{\mathrm{Q} / \mathrm{Z}})$ of $C$. The next result is well-known, but we are unable to find a precise reference for it.

LEMma 2.2. For any complex $C$ of $R$-modules the following conditions hold for any $n \in \mathrm{Z}$

(1) $\mathrm{Z}_{n}\left(C^{+}\right) \cong \operatorname{Hom}_{\mathrm{Z}}\left(C_{-n} / \mathrm{B}_{-n}(C), \mathrm{Q} / \mathrm{Z}\right)=\left(C_{-n} / \mathrm{B}_{-n}(C)\right)^{+}$.

(2) $\mathrm{B}_{n}\left(C^{+}\right) \cong \operatorname{Hom}_{\mathrm{Z}}\left(\mathrm{B}_{-n-1}(C), \mathrm{Q} / \mathrm{Z}\right)=\left(\mathrm{B}_{-n-1}(C)\right)^{+}$.

(3) $\mathrm{H}_{n}\left(C^{+}\right) \cong\left(\mathrm{H}_{-n}(C)\right)^{+}$.

Proof. If $C=\cdots \rightarrow C_{n+1} \stackrel{d_{n+1}}{\longrightarrow} C_{n} \stackrel{d_{n}}{\longrightarrow} C_{n-1} \rightarrow \cdots$, then by Lemma $1.1(3), C^{+}$is

$$
\begin{aligned}
\cdots \rightarrow \operatorname{Hom}_{\mathrm{Z}}\left(C_{-n-1}, \mathrm{Q} / \mathrm{Z}\right) \stackrel{d_{n}^{*}}{\longrightarrow} \operatorname{Hom}_{\mathrm{Z}}\left(C_{-n}, \mathrm{Q} / \mathrm{Z}\right) \\
\stackrel{d_{-n+1}^{*}}{\longrightarrow} \operatorname{Hom}_{\mathrm{Z}}\left(C_{-n+1}, \mathrm{Q} / \mathrm{Z}\right) \rightarrow \cdots
\end{aligned}
$$


with $n$th component $\left(C^{+}\right)_{n}=\operatorname{Hom}\left(C_{-n}, \mathrm{Q} / \mathrm{Z}\right)$, and so

$$
\begin{aligned}
\mathrm{Z}_{n}\left(C^{+}\right) & =\operatorname{Ker}\left(d_{-n+1}^{*}\right)=\left\{f \in \operatorname{Hom}_{\mathrm{Z}}\left(C_{-n}, \mathrm{Q} / \mathrm{Z}\right) \mid f d_{-n+1}=0\right\} \\
& \left.\cong \operatorname{Hom}_{\mathrm{Z}}\left(C_{-n} / \mathrm{B}_{-n}(C)\right), \mathrm{Q} / \mathrm{Z}\right)=\left(C_{-n} / \mathrm{B}_{-n}(C)\right)^{+} \\
\mathrm{B}_{n}\left(C^{+}\right) & =\operatorname{Im}\left(d_{-n}^{*}\right)=\left\{f d_{-n} \mid f \in \operatorname{Hom}_{\mathrm{Z}}\left(C_{-n-1}, \mathrm{Q} / \mathrm{Z}\right)\right\} \\
& \cong \operatorname{Hom}_{\mathrm{Z}}\left(\mathrm{B}_{-n-1}(C), \mathrm{Q} / \mathrm{Z}\right)=\left(\mathrm{B}_{-n-1}(C)\right)^{+}
\end{aligned}
$$

Note that $0 \rightarrow \mathrm{H}_{-n}(C) \rightarrow C_{-n} / \mathrm{B}_{-n}(C) \rightarrow \mathrm{B}_{-n-1}(C) \rightarrow 0$ is exact, thus $0 \rightarrow\left(\mathrm{B}_{-n-1}(C)\right)^{+} \rightarrow\left(C_{-n} / \mathrm{B}_{-n}(C)\right)^{+} \rightarrow\left(\mathrm{H}_{-n}(C)\right)^{+} \rightarrow 0$ is exact. Now it follows easily from the proof above that $\mathrm{H}_{n}\left(C^{+}\right) \cong\left(\mathrm{H}_{-n}(C)\right)^{+}$. This completes the proof.

Corollary 2.3. A complex $F$ is C-E flat in $C$ ( $R$-Mod) if and only if $F^{+}$is $\mathrm{C}-\mathrm{E}$ injective in $C(\operatorname{Mod}-R)$. If $R$ is right coherent, then a complex I of right $R$-modules is C-E injective if and only if $I^{+}$is $\mathrm{C}$-E flat in $C(R$-Mod).

Recall that if $\mathscr{D}$ is a class of objects in an abelian category $\mathscr{A}$ and $X \in \mathscr{A}$, then a $\mathscr{D}$-precover of $X$ is a morphism $f: D \rightarrow X$ with $D \in \mathscr{D}$, such that the triangle

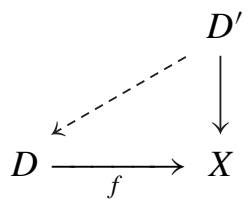

can be completed for each morphism $D^{\prime} \rightarrow X$ with $D^{\prime} \in \mathscr{D}$. A $\mathscr{D}$-precover $f: D \rightarrow X$ is called special if $f$ is epimorphic and $\operatorname{Ext}^{1}(G, \operatorname{Ker}(f))=0$ for all $G \in \mathscr{D}$. If the triangle

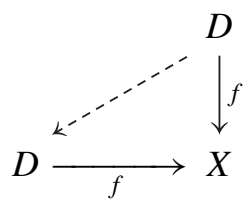

can be completed only by isomorphisms, then $f$ is called a $\mathscr{D}$-cover. (Special) $\mathscr{D}$-preenvelopes and $\mathscr{D}$-envelopes are defined dually.

According to [4, Proposition 7.3], every complex $C$ has a C-E flat cover, which is easily seen epimorphic since any projective complex is clearly C-E flat.

Lemma 2.4. If $F \rightarrow C$ is a $\mathrm{C}$-E flat precover of $C$ with kernel $K$ then the sequence $0 \rightarrow K \rightarrow F \rightarrow C \rightarrow 0$ is $\mathrm{C}$-E exact.

Proof. We note that each $\Sigma^{i} \underline{R}$ is C-E projective, and so it is C-E flat. Then applying the functor $\operatorname{Hom}\left(\Sigma^{i} \underline{R},-\right)$ to the exact sequence $0 \rightarrow K \rightarrow F \rightarrow$ 
$C \rightarrow 0$, we get that $0 \rightarrow \mathrm{Z}_{i}(K) \rightarrow \mathrm{Z}_{i}(F) \rightarrow \mathrm{Z}_{i}(C) \rightarrow 0$ is exact by [4, Proposition 2.1]. Therefore, $0 \rightarrow K \rightarrow F \rightarrow C \rightarrow 0$ is C-E exact.

Lemma 2.5. A complex $F$ is C-E flat in $C$ ( $R$-Mod) if and only if $-\otimes F$ is exact for any short $\mathrm{C}$-E exact sequence of complexes of right $R$-modules.

Proof. Suppose that $F$ is a C-E flat complex and $0 \rightarrow A \rightarrow B \rightarrow C \rightarrow 0$ is a short C-E exact sequence of complexes of right $R$-modules. Then $F=$ $\lim _{i} P_{i}$ with $P_{i}$ C-E projective complexes by [4, Theorem 7.2]. Hence, by $\overrightarrow{\mathrm{Lem} m a s}$ 1.1(1) and 2.1, we get that the sequence $0 \rightarrow A \otimes F \rightarrow B \otimes F \rightarrow$ $C \otimes F \rightarrow 0$ is exact.

Conversely suppose that $-\otimes F$ is exact for any short C-E exact sequence. By Corollary 2.3 we need only to show that $F^{+}=\underline{\operatorname{Hom}}(F, \overline{\mathrm{Q} / \mathrm{Z}})$ is C-E injective in $C$ (Mod- $R$ ). For any complex $A$ of right $R$-modules we let $0 \rightarrow$ $K \rightarrow P \rightarrow A \rightarrow 0$ be a short C-E exact sequence in $C(\operatorname{Mod}-R)$ with $P$ C-E projective (its existence follows from [4, Proposition 5.4]). Then we have the commutative diagram

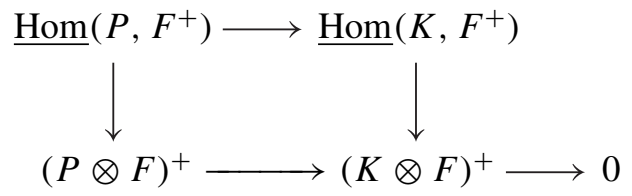

where the vertical arrows are isomorphisms by Lemma 1.1(1). Thus, the morphism $\underline{\operatorname{Hom}}\left(P, F^{+}\right) \rightarrow \underline{\operatorname{Hom}}\left(K, F^{+}\right)$is epic, and so $\operatorname{Hom}\left(P, F^{+}\right) \rightarrow$ $\operatorname{Hom}\left(K, F^{+}\right) \rightarrow 0$ is exact. On the other hand, we get that the sequence $\operatorname{Hom}\left(P, F^{+}\right) \rightarrow \operatorname{Hom}\left(K, F^{+}\right) \rightarrow \overline{\operatorname{Ext}}^{1}\left(A, F^{+}\right) \rightarrow \overline{\operatorname{Ext}}^{1}\left(P, F^{+}\right)$is exact, where $\overline{\operatorname{Ext}}^{1}\left(P, F^{+}\right)=0$ by [4, Theorem 9.4]. This implies that $\overline{\mathrm{Ext}}^{1}\left(A, F^{+}\right)=$ 0 , and so $F^{+}$is C-E injective in $C(\operatorname{Mod}-R)$ by [4, Theorem 9.4].

Now for any complex $C$ we have a left C-E flat resolution $\cdots \rightarrow F_{1} \rightarrow$ $F_{0} \rightarrow C \rightarrow 0$, that is, $F_{0} \rightarrow C$ and $F_{i} \rightarrow K_{i-1}$ are all C-E flat precovers, where $K_{i-1}=\operatorname{Ker}\left(F_{i-1} \rightarrow F_{i-2}\right)$ for all $i \geq 1$ with $F_{-1}=C$. Then by Lemmas 2.4 and 2.5 we see that $F \otimes-$ applied to this resolution gives us an exact sequence for any $\mathrm{C}-\mathrm{E}$ flat complex $F$ in $C(\operatorname{Mod}-R)$. This comment can be used to give us the following result.

TheOREM 2.6. The functor $-\otimes-$ is left balanced on $C(\operatorname{Mod}-R) \times$ $C(R$-Mod) by $\mathrm{CE}($ Flat $-R) \times \mathrm{CE}(R$-Flat), where $R$-Flat (respectively, Flat $R)$ denotes the class of flat (respectively, right) $R$-modules.

REMARK 2.7. By Theorem 2.6 together with the covariant-covariant version of [14, Theorem 2.6], we can compute left derived functors of $X \otimes Y$ either 
using a left C-E flat resolution of $X$ or $Y$. We denote these derived functors by $\overline{\operatorname{Tor}}_{i}(-,-)$. Then it is easy to check the following properties of $\overline{\operatorname{Tor}}_{i}(-,-)$.

(1) $\overline{\operatorname{Tor}}_{0}(-,-)=-\otimes-$.

(2) $\overline{\operatorname{Tor}}_{i}(-, D)=0$ for all $i \geq 1$ and any C-E flat complex $D$ of $R$-modules.

(3) $\overline{\operatorname{Tor}}_{i}(D,-)=0$ for all $i \geq 1$ and any C-E flat complex $D$ of right $R$-modules.

The next result gives some relations between the new functor $\overline{\operatorname{Tor}}_{i}(-,-)$ and the classical one $\operatorname{Tor}_{i}(-,-)$.

Proposition 2.8. Let $C$ be a complex of $R$-modules. Then the following statements are equivalent.

(1) $C$ is exact.

(2) $\operatorname{Tor}_{i}(-, C) \cong \overline{\operatorname{Tor}}_{i}(-, C)$ for all $i \geq 0$.

(3) $\operatorname{Tor}_{1}(-, C) \cong \overline{\operatorname{Tor}}_{1}(-, C)$.

Proof. $(1) \Rightarrow(2)$. Let $C$ be an exact complex and $\cdots \rightarrow F_{2} \rightarrow F_{1} \rightarrow$ $F_{0} \rightarrow C \rightarrow 0$ be a left special flat resolution of $C$, that is, $F_{0} \rightarrow C$ and $F_{i} \rightarrow K_{i-1}$ are all special flat precovers, where $K_{i-1}=\operatorname{Ker}\left(F_{i-1} \rightarrow F_{i-2}\right)$ for all $i \geq 1$ with $F_{-1}=C$. Then $\operatorname{Ext}^{1}\left(F, K_{i}\right)=0$ for any flat complex $F$, and it is easy to see that $K_{i}$ is exact for all $i \geq 0$. Thus it follows from [10, Proposition 4.3.3(1)] and [11, Theorem 3.12] that all $K_{i}$ are C-E cotorsion complexes for $i \geq 0$, and so $\overline{\operatorname{Ext}}^{1}\left(G, K_{i}\right)=0$ for any C-E flat complex $G$ by [4, Theorem 9.4]. We note that the sequence $\cdots \rightarrow F_{2} \rightarrow F_{1} \rightarrow F_{0} \rightarrow C \rightarrow 0$ is $\mathrm{C}$-E exact, then the sequence $\cdots \rightarrow F_{2} \rightarrow F_{1} \rightarrow F_{0} \rightarrow C \rightarrow 0$ is a left C-E flat resolution of $C$, and so we have $\operatorname{Tor}_{i}(D, C) \cong \overline{\operatorname{Tor}}_{i}(D, C)$ for any complex $D$ of right $R$-modules and $i \geq 0$.

$(2) \Rightarrow(3)$ is trivial.

(3) $\Rightarrow$ (1). If $\operatorname{Tor}_{1}(D, C) \cong \overline{\operatorname{Tor}}_{1}(D, C)$ for any complex $D$ of right $R$ modules, then we have $\operatorname{Tor}_{1}\left(\Sigma^{k} \underline{R}, C\right) \cong \overline{\operatorname{Tor}}_{1}\left(\Sigma^{k} \underline{R}, C\right)=0$ by Remark $2.7(3)$, and so

$$
\underline{\operatorname{Ext}}^{1}\left(\Sigma^{k} \underline{R}, C^{+}\right) \cong\left(\operatorname{Tor}_{1}\left(\Sigma^{k} \underline{R}, C\right)\right)^{+}=0
$$

by $\left[10\right.$, Lemma 5.4.2(b)]. Thus $\operatorname{Ext}^{1}\left(\Sigma^{k} \underline{R}, C^{+}\right)=0$, and so $C^{+}$is an exact complex by [5, Remark 5.2]. This implies that $C$ is exact.

Recall that a complex $P$ is finitely generated if, in case $P=\sum_{\lambda \in \Lambda} P_{\lambda}$ with $P_{\lambda}$ subcomplexes of $P$, then there exists a finite subset $F \subseteq \Lambda$ such that $P=\sum_{\lambda \in F} P_{\lambda}$. A complex $Q$ is finitely presented if $Q$ is finitely generated and for any exact sequence of complexes $0 \rightarrow K \rightarrow P \rightarrow Q \rightarrow 0$ with $P$ finitely generated, $K$ is also finitely generated. In fact, a complex $P$ is finitely generated (respectively, presented) if and only if $P$ is bounded (that 
is, $P_{i}=0$ holds for $|i| \gg 0$ ) and each $P_{i}$ is finitely generated (respectively, presented) for $i \in \mathrm{Z}$. According to [6, Definition 2.6], a short exact sequence of complexes $0 \rightarrow S \rightarrow C \rightarrow C / S \rightarrow 0$ is said to be pure, if $0 \rightarrow D \otimes S \rightarrow$ $D \otimes C$ is exact for any (finitely presented) complex $D$ in $C$ (Mod- $R$ ), or equivalently, $\underline{\operatorname{Hom}}(P, C) \rightarrow \underline{\operatorname{Hom}}(P, C / S) \rightarrow 0$ is exact for any finitely presented complex $P$. In this case, we say $S$ a pure subcomplex of $C$.

Lemma 2.9. Every pure subcomplex of a C-E flat complex is C-E flat.

Proof. Let $K \leq F$ be a pure subcomplex of a C-E flat complex $F$. Given a short C-E exact sequence $0 \rightarrow A \rightarrow B \rightarrow C \rightarrow 0$ in $C$ (Mod- $R$ ), we then have the following commutative diagram

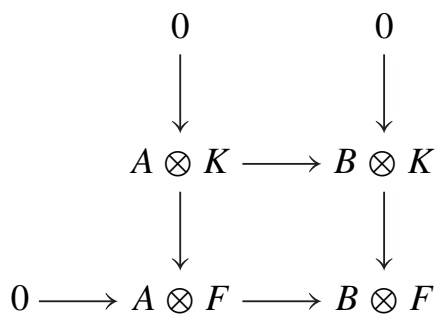

where the bottom row is exact by Lemma 2.5 . Note that all the columns are exact since $K$ is pure in $F$. Then we have that $A \otimes K \rightarrow B \otimes K$ is a monomorphism, and so $K$ is C-E flat by Lemma 2.5.

Using an argument as in the proof of [10, Theorem 5.2.2], we get the following result.

THeOREM 2.10. Let $R$ be a ring. Then the following conditions are equivalent.

(1) $R$ is right coherent.

(2) Every complex has a C-E flat preenvelope.

Proof. (1) $\Rightarrow$ (2). We note that $\mathrm{B}_{i}\left(\prod C_{\alpha}\right) \cong \prod \mathrm{B}_{i}\left(C_{\alpha}\right), \mathrm{Z}_{i}\left(\prod C_{\alpha}\right) \cong$ $\prod \mathrm{Z}_{i}\left(C_{\alpha}\right)$ and $\mathrm{H}_{i}\left(\prod C_{\alpha}\right) \cong \prod \mathrm{H}_{i}\left(C_{\alpha}\right)$ for any family of complexes $\left\{C_{\alpha}\right\}$. Then it is easy to see that under the hypothesis the class of C-E flat complexes is closed under direct products.

Given a complex $C$, we take $\kappa$ an infinite cardinal number such that $\operatorname{Card}(C)$. $\operatorname{Card}(R) \leq \kappa$. Set $S=\{F \in C(R$-Mod $) \mid F$ is C-E flat and $\operatorname{Card}(F) \leq \kappa\}$. Let $\left\{F_{\lambda}\right\}_{\lambda \in \Lambda}$ be a family of representatives of this class with index set $\Lambda$. Let $S_{\lambda}=\operatorname{Hom}\left(C, F_{\lambda}\right)$ for each $\lambda \in \Lambda$ and let $F=\prod_{\lambda \in \Lambda} F_{\lambda}^{S_{\lambda}}$. Now define $f: C \rightarrow F$ so that the composition of $f$ with the projection map $F \rightarrow F_{\lambda}^{S_{\lambda}}$ maps $x \in C_{i}$ to $\left(h_{i}(x)\right)_{h \in S_{\lambda}}$. Then it easy to see that $f: C \rightarrow F$ is a morphism. In the next, we show that $f: C \rightarrow F$ is a C-E flat preenvelope of $C$. Let 
$g: C \rightarrow G$ be a morphism with $G$ a C-E flat complex. By [10, Lemma 5.2.1], the subcomplex $g(C)$ can be enlarged to a pure subcomplex $H \leq G$ with $\operatorname{Card}(H) \leq \kappa$. Since $H$ is C-E flat by Lemma 2.9, $H$ is isomorphic to one of the $F_{\lambda}$. By construction of the morphism $f$, it is not hard to show that $g$ can be factored through $f$, as desired.

(2) $\Rightarrow(1)$. Let $M$ be an $R$-module and $\phi: \bar{M} \rightarrow F$ be a C-E flat preenvelope of $\bar{M}$. Then one can check easily that $\phi_{1}: M \rightarrow F_{1}$ is a flat preenvelope of $M$, and so $R$ is right coherent by [7, Proposition 6.5.1].

In the end of this section, we give another characterization of C-E flat complexes.

Proposition 2.11. For a complex $F$, the following conditions are equivalent.

(1) $F$ is C-E flat.

(2) Every short C-E exact sequence $0 \rightarrow K \rightarrow P \rightarrow F \rightarrow 0$ is pure.

(3) There exists a pure exact sequence $0 \rightarrow K \rightarrow P \rightarrow F \rightarrow 0$ such that $P$ is C-E projective (C-E flat).

Proof. (1) $\Rightarrow$ (2). Let $0 \rightarrow K \rightarrow P \rightarrow F \rightarrow 0$ be a short C-E exact sequence and let $C$ be a complex of right $R$-modules. If $Q \rightarrow C$ is a C-E projective precover of $C$ then we have a C-E exact sequence $0 \rightarrow L \rightarrow Q \rightarrow$ $C \rightarrow 0$ by [4, Proposition 5.4]. Consider the following commutative diagram



Since every C-E projective complex is C-E flat, we get that the right-hand column and the middle row in the diagram above are exact by Lemma 2.5 . Thus, we get that $0 \rightarrow C \otimes K \rightarrow C \otimes P \rightarrow C \otimes F \rightarrow 0$ is exact by the snake lemma. Hence the $\mathrm{C}$-E exact sequence $0 \rightarrow K \rightarrow P \rightarrow F \rightarrow 0$ is pure.

(2) $\Rightarrow$ (3) follows from [4, Proposition 5.4]. 
(3) $\Rightarrow$ (1). Let $0 \rightarrow K \rightarrow P \rightarrow F \rightarrow 0$ be a pure exact sequence with $P$ C-E projective (C-E flat), and let $0 \rightarrow A \rightarrow B \rightarrow C \rightarrow 0$ be a C-E exact sequence in $C(\operatorname{Mod}-R)$. Now Consider the following commutative diagram

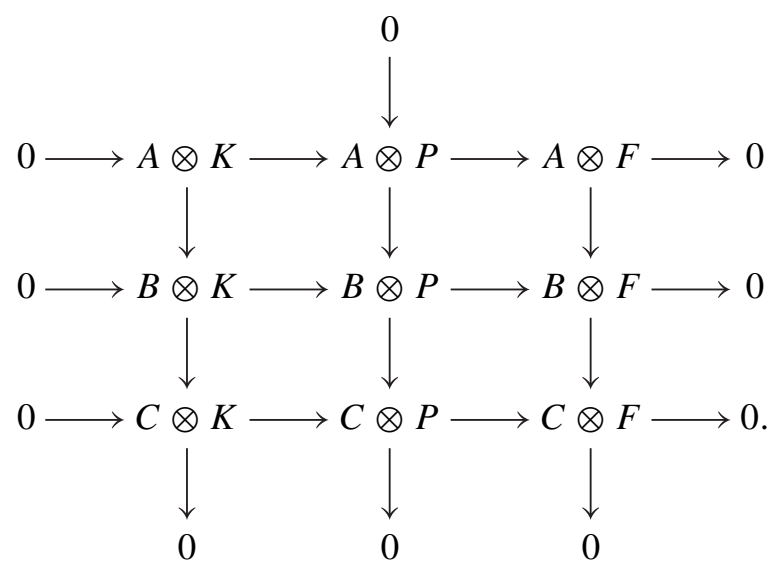

Since all the rows and the middle column in the diagram above are exact by hypothesis, we get by the snake lemma that the right-hand column is exact. Thus $F$ is C-E flat by Lemma 2.5.

Corollary 2.12. Let $0 \rightarrow X \rightarrow Y \rightarrow Z \rightarrow 0$ be a $\mathrm{C}$-E exact sequence with $Z \mathrm{C}-\mathrm{E}$ flat. Then $X$ is $\mathrm{C}-\mathrm{E}$ flat if and only if $Y$ is $\mathrm{C}-\mathrm{E}$ flat.

Proof. Let $0 \rightarrow A \rightarrow B \rightarrow C \rightarrow 0$ be a $\mathrm{C}$-E exact sequence in $C($ Mod- $R$ ). Then we get that all the rows in the following commutative diagram are exact by Proposition 2.11, and the right-hand column is exact by Lemma 2.5 since $Z$ is C-E flat.

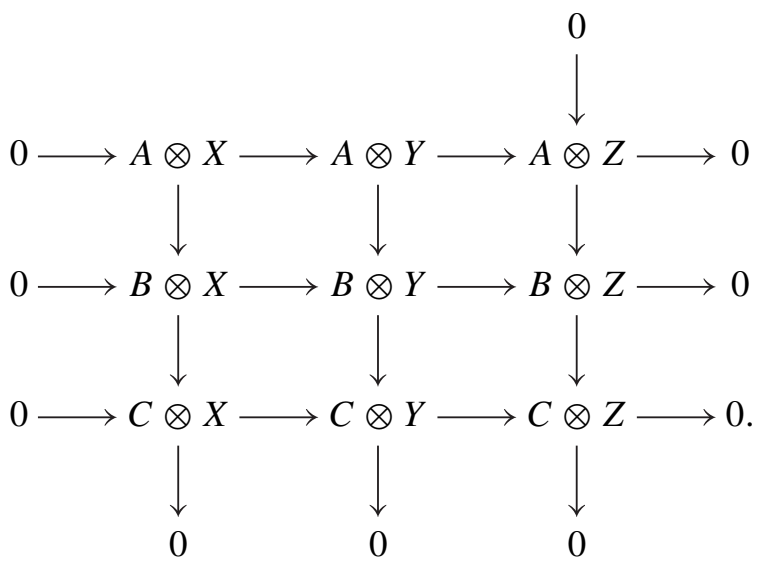

Thus the above diagram implies that $0 \rightarrow A \otimes Y \rightarrow B \otimes Y \rightarrow C \otimes Y \rightarrow 0$ 
is exact if and only if $0 \rightarrow A \otimes X \rightarrow B \otimes X \rightarrow C \otimes X \rightarrow 0$ is exact. Hence $Y$ is C-E flat if and only if $X$ is C-E flat by Lemma 2.5 .

\section{C-E Gorenstein flat complexes}

We have already defined a C-E Gorenstein flat complex in Definition 1.4. But we show that over right coherent rings one can also use a modification of Definition 1.3 to define such a complex. We start with the following.

Lemma 3.1. Let $R$ be a right coherent ring and $M$ a Gorenstein flat $R$ module. Then any flat preenvelope $f: M \rightarrow F$ of $M$ is a monomorphism and Coker $(f)$ is a Gorenstein flat $R$-module.

Proof. By [7, Proposition 6.5.1], $M$ has a flat preenvelope $f: M \rightarrow F$. Since $M$ is a Gorenstein flat $R$-module, there exists an exact sequence $0 \rightarrow$ $M \stackrel{\alpha}{\longrightarrow} F_{-1}$ with $F_{-1}$ flat. Thus $f$ must be a monomorphism since there exists a homomorphism $g: F \rightarrow F_{-1}$ such that $g f=\alpha$. Hence, we have the exact sequence $0 \rightarrow M \stackrel{f}{\rightarrow} F \rightarrow N \rightarrow 0$, where $N=\operatorname{Coker}(f)$. Let $I$ be any injective right $R$-module. Then we have the following commutative diagram

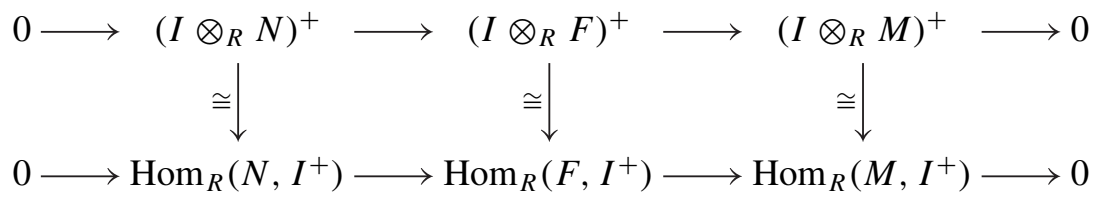

where the bottom row is exact since $f: M \rightarrow F$ is a flat preenvelope of $M$ and $I^{+}$is flat. So the top row is exact too. This yields the exactness of $0 \rightarrow I \otimes_{R} M \rightarrow I \otimes_{R} F \rightarrow I \otimes_{R} N \rightarrow 0$. Thus $\operatorname{Tor}_{1}^{R}(I, N)=0$, and hence $N$ is Gorenstein flat by [13, Proposition 3.8].

It was shown by Enochs [4, Theorem 8.5] that a complex $G$ is C-E Gorenstein injective if and only if there exists a C-E exact sequence $\cdots \rightarrow I_{2} \rightarrow$ $I_{1} \rightarrow I_{0} \rightarrow I_{-1} \rightarrow I_{-2} \rightarrow \cdots$ of C-E injective complexes with $G=$ $\operatorname{Ker}\left(I_{-1} \rightarrow I_{-2}\right)$, such that it remains exact after applying $\operatorname{Hom}(J,-)$ for any C-E injective complex $J$.

In the next, we focus on Cartan-Eilenberg Gorenstein flat complexes and we show that over right coherent rings such complexes can be gotten by a so called complete Cartan-Eilenberg flat resolution.

Definition 3.2. For a complex $G \in C(R$-Mod), by a complete C-E flat resolution of $G$ we mean a C-E exact sequence $\cdots \rightarrow F_{2} \rightarrow F_{1} \rightarrow F_{0} \rightarrow$ $F_{-1} \rightarrow F_{-2} \rightarrow \cdots$ of C-E flat complexes with $G=\operatorname{Ker}\left(F_{-1} \rightarrow F_{-2}\right)$, such that it remains exact after applying $I \otimes-$ for any C-E injective complex $I$ of right $R$-modules. 
In the following we use the symbol $R$-Gorflat to stand for the class of Gorenstein flat $R$-modules.

LeMma 3.3. Let $R$ be a right coherent ring. Then a complex $G$ in $C$ ( $R$-Mod) is such that $G$ and $G / \mathrm{B}(G)$ are in $C\left(R\right.$-Gorflat) if and only if $G^{+}$is C-E Gorenstein injective in $C$ (Mod- $R$ ).

Proof. Assume that $G$ and $G / \mathrm{B}(G)$ are in $C$ (R-Gorflat). Then all right $R$-modules $\operatorname{Hom}_{\mathrm{Z}}\left(G_{-n}, \mathrm{Q} / \mathrm{Z}\right)$ and $\operatorname{Hom}_{\mathrm{Z}}\left(G_{-n} / \mathrm{B}_{-n}(G), \mathrm{Q} / \mathrm{Z}\right)$ are Gorenstein injective by [13, Theorem 3.6], but $\operatorname{Hom}_{\mathrm{Z}}\left(G_{-n} / \mathrm{B}_{-n}(G), \mathrm{Q} / \mathrm{Z}\right) \cong \mathrm{Z}_{n}\left(G^{+}\right)$by Lemma 2.2, and clearly $\operatorname{Hom}_{\mathbf{Z}}\left(G_{-n}, \mathrm{Q} / \mathrm{Z}\right)=\left(G^{+}\right)_{n}$. Now using the exact sequences $0 \rightarrow \mathrm{Z}_{n}\left(G^{+}\right) \rightarrow\left(G^{+}\right)_{n} \rightarrow \mathrm{B}_{n-1}\left(G^{+}\right) \rightarrow 0$ and $0 \rightarrow \mathrm{B}_{n}\left(G^{+}\right) \rightarrow$ $\mathrm{Z}_{n}\left(G^{+}\right) \rightarrow \mathrm{H}_{n}\left(G^{+}\right) \rightarrow 0$, we get by [13, Theorem 2.6] that all right $R$ modules $\mathrm{B}_{n}\left(G^{+}\right)$and $\mathrm{H}_{n}\left(G^{+}\right)$are Gorenstein injective, and so $G^{+}$is C-E Gorenstein injective in $C$ (Mod- $R$ ) by [4, Theorem 8.5].

Conversely suppose $G^{+}$is C-E Gorenstein injective in $C$ (Mod- $R$ ). Then by $\left[4\right.$, Theorem 8.5] we get that each $\left(G^{+}\right)_{n}=\operatorname{Hom}_{\mathrm{Z}}\left(G_{-n}, \mathrm{Q} / \mathrm{Z}\right)$, and $\mathrm{Z}_{n}\left(G^{+}\right)$, which is isomorphic to $\operatorname{Hom}_{\mathrm{Z}}\left(G_{-n} / \mathrm{B}_{-n}(G), \mathrm{Q} / \mathrm{Z}\right)$ by Lemma 2.2, are Gorenstein injective, and so $G_{-n}$ and $G_{-n} / \mathrm{B}_{-n}(G)$ are Gorenstein flat by [13, Theorem 3.6]. This proves that $G$ and $G / \mathrm{B}(G)$ are in $C$ (R-Gorflat).

Remark 3.4. Let $f: X \rightarrow Y$ be a morphism of complexes. As one has $\delta_{i}^{Y} f_{i}=f_{i-1} \delta_{i}^{X}$ for all $i \in \mathrm{Z}$, there is an inclusion $f(\mathrm{~B}(X)) \subseteq \mathrm{B}(Y)$. It follows that $f$ induces a morphism of complexes $\bar{f}: X / \mathrm{B}(X) \rightarrow Y / \mathrm{B}(Y)$, which is given by the assignment $x+\mathrm{B}_{i}(X) \mapsto f_{i}(x)+\mathrm{B}_{i}(Y)$ for any $x \in X_{i}$. With this definition one can check easily that $C \rightarrow C / \mathrm{B}(C)$ is a right exact functor.

THeOREM 3.5. Let $R$ be a right coherent ring and $G$ be a complex in $C(R$-Mod). Then the following conditions are equivalent.

(1) $\mathrm{B}(G)$ and $\mathrm{H}(G)$ are in $C$ ( $R$-Gorflat).

(2) $G$ and $G / \mathrm{B}(G)$ are in $C$ ( $R$-Gorflat).

(3) $G$ has a complete C-E flat resolution.

(4) $G$ is C-E Gorenstein flat.

Proof. (1) $\Rightarrow$ (2). Since $\mathrm{B}_{m}(G)$ and $\mathrm{H}_{m}(G)$ are Gorenstein flat in $R$-Mod, and the sequences $0 \rightarrow \mathrm{B}_{m}(G) \rightarrow \mathrm{Z}_{m}(G) \rightarrow \mathrm{H}_{m}(G) \rightarrow 0$ and $0 \rightarrow$ $\mathrm{Z}_{m}(G) \rightarrow G_{m} \rightarrow \mathrm{B}_{m-1}(G) \rightarrow 0$ are exact for all $m \in \mathrm{Z}$, we get from [13, Theorem 3.7] that $G_{m}$ are Gorentein flat in $R$-Mod for all $m \in Z$. For the same argument we get that $G_{m} / \mathrm{B}_{m}(G)$ is Gorentein flat since the sequence $0 \rightarrow \mathrm{H}_{m}(G) \rightarrow G_{m} / \mathrm{B}_{m}(G) \rightarrow \mathrm{B}_{m-1}(G) \rightarrow 0$ is exact.

(2) $\Rightarrow$ (1) can be proved similarly.

(2) $\Rightarrow(3)$. By Theorem 2.10, $G$ has a C-E flat preenvelope $\alpha: G \rightarrow F_{-1}$. Suppose that $F$ is a flat $R$-module. Then $\Sigma^{m} \bar{F}$ is C-E flat for any $m \in \mathrm{Z}$, and 
so $\operatorname{Hom}\left(F_{-1}, \Sigma^{m} \bar{F}\right) \rightarrow \operatorname{Hom}\left(G, \Sigma^{m} \bar{F}\right) \rightarrow 0$ is exact. This implies that

$$
\operatorname{Hom}\left(\left(F_{-1}\right)_{m}, F\right) \rightarrow \operatorname{Hom}\left(G_{m}, F\right) \rightarrow 0
$$

is exact by [4, Proposition 2.1]. Thus $\alpha_{m}: G_{m} \rightarrow\left(F_{-1}\right)_{m}$ is a flat preenvelope of $G_{m}$, and so $\alpha_{m}$ is a monomorphism and $\operatorname{Coker}\left(\alpha_{m}\right)$ is a Gorenstein flat $R$-module by Lemma 3.1 since $G_{m}$ is Gorenstein flat. Hence, we have an exact sequence of complexes $0 \rightarrow G \stackrel{\alpha}{\longrightarrow} F_{-1} \rightarrow L_{-1} \rightarrow 0$, where $L_{-1}=$ $\operatorname{Coker}(\alpha)$ is in $C$ (R-Gorflat). Since the functor $C \rightarrow C / B(C)$ is right exact by Remark 3.4, we get that $G / B(G) \rightarrow F_{-1} / B\left(F_{-1}\right) \rightarrow L_{-1} / B\left(L_{-1}\right) \rightarrow 0$ is exact. Now for any flat $R$-module $F$, applying the functor $\operatorname{Hom}\left(-, \Sigma^{m} \underline{F}\right)$ to the exact sequence $0 \rightarrow G \stackrel{\alpha}{\longrightarrow} F_{-1} \rightarrow L_{-1} \rightarrow 0$, we get by [4, Proposition 2.1] that the sequence

$$
\begin{aligned}
& 0 \rightarrow \operatorname{Hom}\left(\left(L_{-1}\right)_{m} / \mathrm{B}_{m}\left(L_{-1}\right), F\right) \rightarrow \operatorname{Hom}\left(\left(F_{-1}\right)_{m} / \mathrm{B}_{m}\left(F_{-1}\right), F\right) \\
& \rightarrow \operatorname{Hom}\left(G_{m} / \mathrm{B}_{m}(G), F\right) \rightarrow 0
\end{aligned}
$$

is exact since $\Sigma^{m} \underline{F}$ is C-E flat and $\alpha: G \rightarrow F_{-1}$ is a C-E flat preenvelope of $G$. This implies that $G_{m} / \mathrm{B}_{m}(G) \rightarrow\left(F_{-1}\right)_{m} / \mathrm{B}_{m}\left(F_{-1}\right)$ is a flat preenvelope of $G_{m} / \mathrm{B}_{m}(G)$ since $F_{-1}$ is C-E flat. Thus $G_{m} / \mathrm{B}_{m}(G) \rightarrow\left(F_{-1}\right)_{m} / \mathrm{B}_{m}\left(F_{-1}\right)$ is a monomorphism and its cokernel $\left(L_{-1}\right)_{m} / \mathrm{B}_{m}\left(L_{-1}\right)$ is Gorenstein flat by Lemma 3.1 since $G_{m} / \mathrm{B}_{m}(G)$ is Gorenstein flat. Hence, the sequence $0 \rightarrow$ $G / B(G) \rightarrow F_{-1} / B\left(F_{-1}\right) \rightarrow L_{-1} / B\left(L_{-1}\right) \rightarrow 0$ is exact with $L_{-1} / B\left(L_{-1}\right)$ in $C(R$-Gorflat). Therefore, the sequence

$$
0 \rightarrow G \stackrel{\alpha}{\longrightarrow} F_{-1} \rightarrow L_{-1} \rightarrow 0
$$

is $\mathrm{C}$-E exact.

In the following, we show that the C-E exact sequence $(*)$ remains exact after applying $I \otimes-$ for any C-E injective complex $I$ of right $R$-modules. Let $I$ be any C-E injective complex of right $R$-modules. Then we have that $I^{+}$is C-E flat by Corollary 2.3. Consider the following commutative diagram

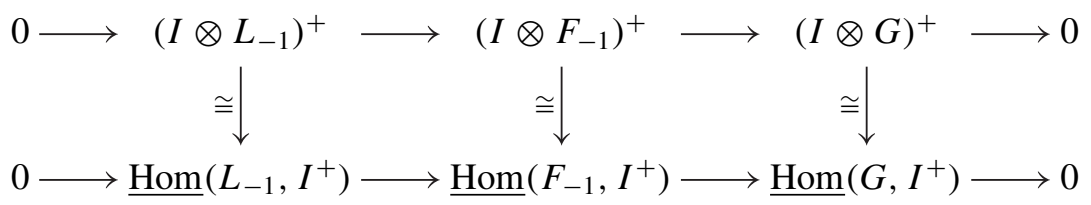

where the vertical isomorphisms are obtained directly by Lemma 1.1(1). Note that the bottom row in the diagram above is exact since $\alpha: G \rightarrow F_{-1}$ is a C-E flat preenvelope of $G$. So the top row is also exact. This means $0 \rightarrow$ $I \otimes G \rightarrow I \otimes F_{-1} \rightarrow I \otimes L_{-1} \rightarrow 0$ is exact. Therefore, the sequence $(*)$ remains exact after applying $I \otimes-$ for any C-E injective complex $I$ of right 
$R$-modules. Using the same procedure we can construct a $\mathrm{C}$-E exact sequence of complexes

$$
0 \rightarrow G \rightarrow F_{-1} \rightarrow F_{-2} \rightarrow \cdots
$$

such that each $F_{i}$ is $\mathrm{C}$-E flat and it remains exact after applying $I \otimes-$ for any $\mathrm{C}$-E injective complex $I$ of right $R$-modules.

Suppose that the sequence

$$
\cdots \rightarrow F_{2} \rightarrow F_{1} \rightarrow F_{0} \rightarrow G \rightarrow 0
$$

is a left C-E flat resolution of $G$. Then we break it into short exact sequences, and we need only to show that all the sequences remain exact after applying $I \otimes-$ for any $\mathrm{C}$-E injective complex $I$ of right $R$-modules. First consider the short exact sequence $0 \rightarrow K_{1} \rightarrow F_{0} \rightarrow G \rightarrow 0$, where $K_{1}=\operatorname{Ker}\left(F_{0} \rightarrow\right.$ $G)$. Then it is C-E exact by Lemma 2.4. Let $I$ be any C-E injective complex of right $R$-modules. Then by [4, Lemmas 9.1 and 9.2] $\overline{\mathrm{Ext}}^{1}(I, X)=$ 0 for any C-E Gorenstein injective complex $X$ since $I$ can be written as $\left(\oplus_{k \in \mathrm{Z}} \Sigma^{k} \overline{E_{k}}\right) \bigoplus\left(\oplus_{k \in \mathrm{Z}} \Sigma^{k} E_{k}^{\prime}\right)$ where $E_{k}, E_{k}^{\prime}$ are injective $R$-modules, and so $\overline{\operatorname{Ext}}^{1}\left(I, \Sigma^{-m} G^{+}\right)=0$ for any $m \in \mathrm{Z}$ since $G^{+}$is C-E Gorenstein injective by Lemma 3.3. Note that the sequence $0 \rightarrow \Sigma^{-m} G^{+} \rightarrow \Sigma^{-m} F_{0}^{+} \rightarrow \Sigma^{-m} K_{1}^{+} \rightarrow$ 0 is C-E exact by Lemma 2.2 , then the sequence $0 \rightarrow \operatorname{Hom}\left(I, \Sigma^{-m} G^{+}\right) \rightarrow$ $\operatorname{Hom}\left(I, \Sigma^{-m} F_{0}^{+}\right) \rightarrow \operatorname{Hom}\left(I, \Sigma^{-m} K_{1}^{+}\right) \rightarrow 0$ is exact. This implies that

$$
0 \rightarrow \underline{\operatorname{Hom}}\left(I, G^{+}\right) \rightarrow \underline{\operatorname{Hom}}\left(I, F_{0}^{+}\right) \rightarrow \underline{\operatorname{Hom}}\left(I, K_{1}^{+}\right) \rightarrow 0
$$

is exact, and so $0 \rightarrow(I \otimes G)^{+} \rightarrow\left(I \otimes F_{0}\right)^{+} \rightarrow\left(I \otimes K_{1}\right)^{+} \rightarrow 0$ is exact by Lemma 1.1(1). Thus $0 \rightarrow I \otimes K_{1} \rightarrow I \otimes F_{0} \rightarrow I \otimes G \rightarrow 0$ is exact, that is, the sequence $0 \rightarrow K_{1} \rightarrow F_{0} \rightarrow G \rightarrow 0$ remains exact after applying $I \otimes-$. Note that the sequence $0 \rightarrow K_{1} \rightarrow F_{0} \rightarrow G \rightarrow 0$ is C-E exact, then one can check that $K_{1}$ and $K_{1} / B\left(K_{1}\right)$ are in $C$ (R-Gorflat) by [13, Theorem 3.7]. Thus, we can continuously use the same method to the other short exact sequences and get that the sequence $(b b)$ remains exact after applying $I \otimes-$ for any $\mathrm{C}$-E injective complex $I$ of right $R$-modules. Now assemble the two sequences (b) and (bb), we get a complete C-E flat resolution of $G$.

(3) $\Rightarrow(2)$. Suppose that the sequence

$$
\cdots \rightarrow F_{2} \rightarrow F_{1} \rightarrow F_{0} \rightarrow F_{-1} \rightarrow F_{-2} \rightarrow \cdots
$$

is a complete $\mathrm{C}$-E flat resolution with $G=\operatorname{Ker}\left(F_{-1} \rightarrow F-2\right)$, and $I$ is a C-E injective complex of right $R$-modules. Then the sequence

$$
\cdots \rightarrow I \otimes F_{2} \rightarrow I \otimes F_{1} \rightarrow I \otimes F_{0} \rightarrow I \otimes F_{-1} \rightarrow I \otimes F_{-2} \rightarrow \cdots
$$


is exact, and so the sequence

$$
\cdots \rightarrow\left(I \otimes F_{-2}\right)^{+} \rightarrow\left(I \otimes F_{-1}\right)^{+} \rightarrow\left(I \otimes F_{0}\right)^{+} \rightarrow\left(I \otimes F_{1}\right)^{+} \rightarrow \cdots
$$

is exact. By Lemma 1.1(1), we get that the sequence

$$
\begin{aligned}
\cdots \rightarrow \underline{\operatorname{Hom}}\left(I, F_{-2}^{+}\right) \rightarrow \underline{\operatorname{Hom}}\left(I, F_{-1}^{+}\right) & \\
& \rightarrow \underline{\operatorname{Hom}}\left(I, F_{0}^{+}\right) \rightarrow \underline{\operatorname{Hom}}\left(I, F_{1}^{+}\right) \rightarrow \cdots
\end{aligned}
$$

is exact. This implies that the sequence

$$
\begin{aligned}
\cdots \rightarrow \operatorname{Hom}\left(I, F_{-2}^{+}\right) \rightarrow \operatorname{Hom}\left(I, F_{-1}^{+}\right) & \\
& \rightarrow \operatorname{Hom}\left(I, F_{0}^{+}\right) \rightarrow \operatorname{Hom}\left(I, F_{1}^{+}\right) \rightarrow \cdots
\end{aligned}
$$

is exact. We note that the sequence

$$
\cdots \rightarrow F_{-2}^{+} \rightarrow F_{-1}^{+} \rightarrow F_{0}^{+} \rightarrow F_{1}^{+} \rightarrow F_{2}^{+} \rightarrow \cdots
$$

is C-E exact, $G^{+}=\operatorname{Ker}\left(F_{0}^{+} \rightarrow F_{1}^{+}\right)$, and each $F_{i}^{+}$is C-E injective by Corollary 2.3. Then $G^{+}$is C-E Gorenstein injective by [4, Theorem 8.5], and hence we get the desired result by Lemma 3.3.

$(1) \Leftrightarrow(4)$ is obvious.

For an $R$-module $M$, the Gorenstein flat dimension, $\operatorname{Gfd}(M)$, is defined by using a resolution by Gorenstein flat $R$-modules, see [13]. Similarly, we give the following definition.

Definition 3.6. The C-E Gorenstein flat dimension, CE-Gfd $(C)$, of a complex $C$ is defined as CE-Gfd $(C)=\inf \{n \mid$ there exists a C-E exact sequence $0 \rightarrow X_{n} \rightarrow X_{n-1} \rightarrow \cdots \rightarrow X_{0} \rightarrow C \rightarrow 0$ with each $X_{i}$ C-E Gorenstein flat $\}$. If no such $n$ exists, set CE-Gfd $(C)=\infty$.

Proposition 3.7. Let $R$ be a right coherent ring and $C$ be a complex of $R$-modules. Then CE-Gfd $(C)=\sup \left\{\operatorname{Gfd}\left(\mathrm{H}_{i}(C)\right), \operatorname{Gfd}\left(\mathrm{B}_{i}(C)\right) \mid i \in \mathrm{Z}\right\}$.

Proof. If $\sup \left\{\operatorname{Gfd}\left(\mathrm{H}_{i}(C)\right), \operatorname{Gfd}\left(\mathrm{B}_{i}(C)\right) \mid i \in \mathrm{Z}\right\}=\infty$, then

$$
\text { CE-Gfd }(C) \leq \sup \left\{\operatorname{Gfd}\left(\mathrm{H}_{i}(C)\right), \operatorname{Gfd}\left(\mathrm{B}_{i}(C)\right) \mid i \in \mathrm{Z}\right\} .
$$

So naturally we may assume that $\sup \left\{\operatorname{Gfd}\left(\mathrm{H}_{i}(C)\right), \operatorname{Gfd}\left(\mathrm{B}_{i}(C)\right) \mid i \in \mathrm{Z}\right\}=n$ is finite. Consider the C-E exact sequence

$$
0 \rightarrow K_{n} \rightarrow F_{n-1} \rightarrow \cdots \rightarrow F_{1} \rightarrow F_{0} \rightarrow C \rightarrow 0,
$$

where each $F_{j}$ is C-E flat. Then we have two exact sequences

$$
0 \rightarrow H\left(K_{n}\right) \rightarrow H\left(F_{n-1}\right) \rightarrow \cdots \rightarrow H\left(F_{1}\right) \rightarrow H\left(F_{0}\right) \rightarrow H(C) \rightarrow 0
$$


and

$$
0 \rightarrow B\left(K_{n}\right) \rightarrow B\left(F_{n-1}\right) \rightarrow \cdots \rightarrow B\left(F_{1}\right) \rightarrow B\left(F_{0}\right) \rightarrow B(C) \rightarrow 0,
$$

and so $\mathrm{H}_{i}\left(K_{n}\right)$ and $\mathrm{B}_{i}\left(K_{n}\right)$ are Gorenstein flat for all $i \in \mathrm{Z}$ by [13, Theorem 3.14]. Now, by Theorem 3.5, $K_{n}$ is a C-E Gorenstein flat complex. This shows that $\mathrm{CE}-\mathrm{Gfd}(C) \leq \sup \left\{\operatorname{Gfd}\left(\mathrm{H}_{i}(C)\right), \operatorname{Gfd}\left(\mathrm{B}_{i}(C)\right) \mid i \in \mathrm{Z}\right\}$.

Next we will show that $\sup \left\{\operatorname{Gfd}\left(\mathrm{H}_{i}(C)\right), \operatorname{Gfd}\left(\mathrm{B}_{i}(C)\right) \mid i \in \mathrm{Z}\right\} \leq$ $\mathrm{CE}-\mathrm{Gfd}(C)$. Naturally, we may assume that CE-Gfd $(C)=n$ is finite. Then there exists a C-E exact sequence of complexes $0 \rightarrow G_{n} \rightarrow G_{n-1} \rightarrow \cdots \rightarrow$ $G_{1} \rightarrow G_{0} \rightarrow C \rightarrow 0$ such that each $G_{j}$ is C-E Gorenstein flat. Now since $\mathrm{H}_{i}\left(G_{j}\right)$ and $\mathrm{B}_{i}\left(G_{j}\right)$ are Gorenstein flat modules for all $i \in \mathrm{Z}$ and all $j=0,1, \cdots, n$, we get that $\operatorname{Gfd}\left(\mathrm{H}_{i}(C)\right) \leq n$ and $\operatorname{Gfd}\left(\mathrm{B}_{i}(C)\right) \leq n$ for all $i \in \mathrm{Z}$, and so $\sup \left\{\operatorname{Gfd}\left(\mathrm{H}_{i}(C)\right), \operatorname{Gfd}\left(\mathrm{B}_{i}(C)\right) \mid i \in \mathrm{Z}\right\} \leq n=\mathrm{CE}-\mathrm{Gfd}(C)$, as desired.

The notion of a cotorsion pair was first introduced by Salce in [16] and later rediscovered by Enochs and Jenda [7], and Göbel and Trlifaj [12]. Cotorsion pairs are homologically useful if they are complete. For definitions of undefined terms see [7] and [12]. There the definitions and results were for modules. But it is straightforward to modify them to apply to complexes.

LeMma 3.8. Suppose that $(\mathscr{A}, \mathscr{B})$ is a hereditary and complete cotorsion pair in $R$-Mod and $0 \rightarrow X_{1} \rightarrow X_{2} \rightarrow X_{3} \rightarrow 0$ is a short exact sequence of $R$-modules. If $f_{i}: A_{i} \rightarrow X_{i}$ is a special $\mathscr{A}$-precover of $X_{i}$ for $i=1$ and 3 , then there exists a commutative diagram

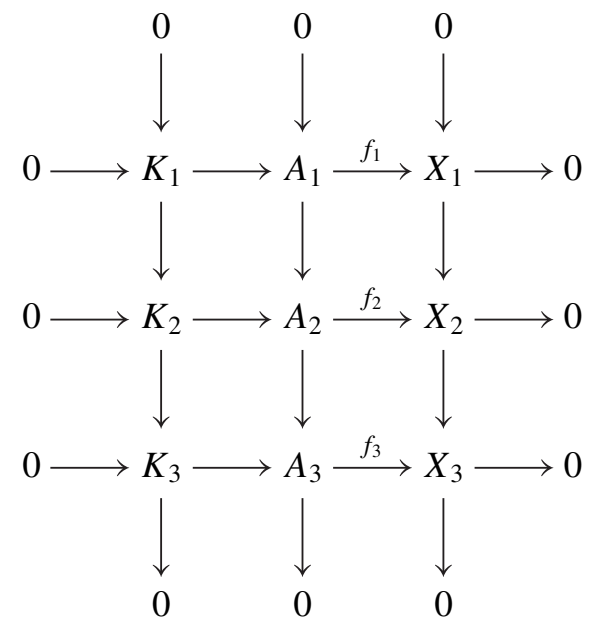

with exact rows and columns such that $f_{2}: A_{2} \longrightarrow X_{2}$ is a special $\mathscr{A}$-precover of $X_{2}$, where $K_{i}=\operatorname{Ker}\left(f_{i}\right)$ for $i=1,2,3$. 
Proof. It follows from [1, Theorem 3.1].

By [4, Theorem 9.4], $(\mathrm{CE}(\mathscr{A}), \mathrm{CE}(\mathscr{B}))$ forms a hereditary cotorsion pair in $C\left(R\right.$-Mod) relative to $\overline{\operatorname{Ext}}^{1}(-,-)$ whenever $(\mathscr{A}, \mathscr{B})$ is a hereditary cotorsion pair in $R$-Mod. Furthermore, we have the following result.

THEOREM 3.9. Let $(\mathscr{A}, \mathscr{B})$ be a hereditary cotorsion pair in $R$-Mod. If $(\mathscr{A}, \mathscr{B})$ is complete then the cotorsion pair $(\mathrm{CE}(\mathscr{A}), \mathrm{CE}(\mathscr{B}))$ in $C(R-\mathrm{Mod})$ relative to $\overline{\mathrm{Ext}}^{1}(-,-)$ is complete.

Proof. Let $C$ be any complex. Then $\mathrm{B}_{i}(C)$ and $\mathrm{H}_{i}(C)$ have special $\mathscr{A}$ precovers since $(\mathscr{A}, \mathscr{B})$ is complete. Let $f_{i}: D_{i} \rightarrow \mathrm{B}_{i}(C)$ be a special $\mathscr{A}$ precover of $\mathrm{B}_{i}(C)$, and $h_{i}: D_{i}^{\prime} \rightarrow \mathrm{H}_{i}(C)$ be a special $\mathscr{A}$-precover of $\mathrm{H}_{i}(C)$. Then using the exact sequence $0 \rightarrow \mathrm{B}_{i}(C) \rightarrow \mathrm{Z}_{i}(C) \rightarrow \mathrm{H}_{i}(C) \rightarrow 0$ and Lemma 3.8 we can construct a special $\mathscr{A}$-precover $\widetilde{f}_{i}: \widetilde{D}_{i} \rightarrow \mathrm{Z}_{i}(C)$ of $\mathrm{Z}_{i}(C)$ such that the following diagram

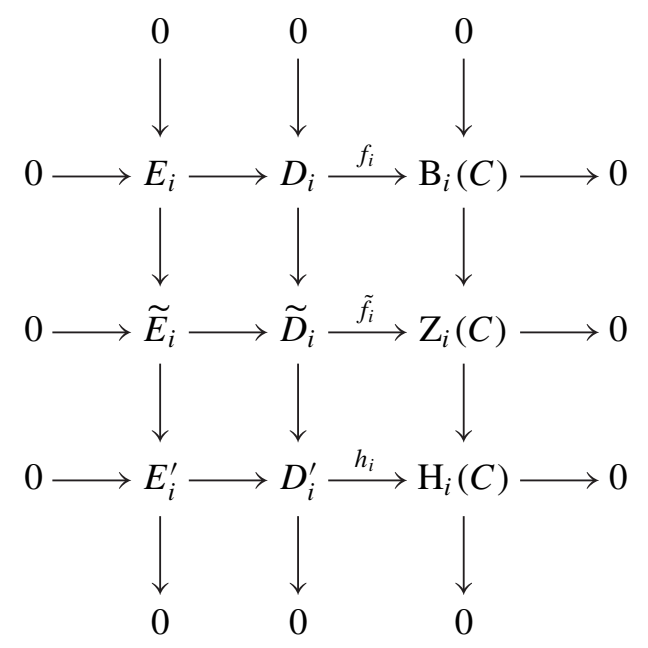

is commutative and each row and column are exact. Using Lemma 3.8 together with the special $\mathscr{A}$-precover $\widetilde{f}_{i}: \widetilde{D}_{i} \rightarrow \mathrm{Z}_{i}(C)$ of $\mathrm{Z}_{i}(C)$ and the given special $\mathscr{A}$-precover $f_{i-1}: D_{i-1} \rightarrow \mathrm{B}_{i-1}(C)$ of $\mathrm{B}_{i-1}(C)$ and the exact sequence $0 \rightarrow \mathrm{Z}_{i}(C) \rightarrow C_{i} \rightarrow \mathrm{B}_{i-1}(C) \rightarrow 0$ we can construct a special $\mathscr{A}$-precover $\phi_{i}: G_{i} \rightarrow C_{i}$ of $C_{i}$ such that the following diagram 


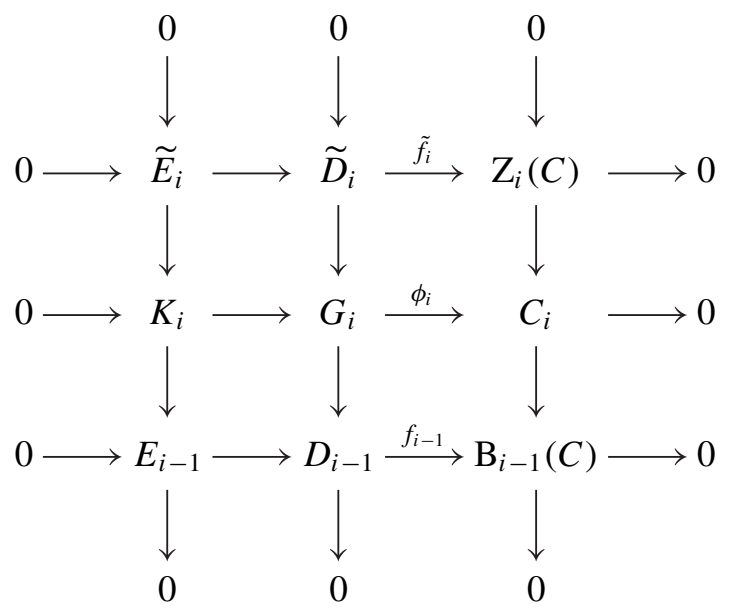

is commutative and each row and column are exact. By construction above we have the following commutative diagram

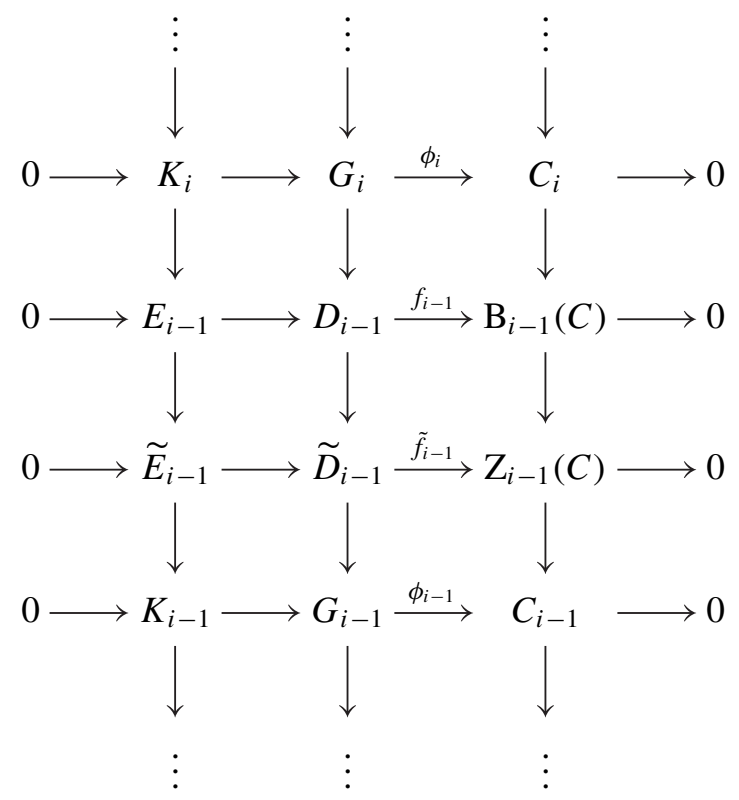

with exact rows. This yields an exact sequence $0 \rightarrow K \rightarrow G \stackrel{\phi}{\longrightarrow} C \rightarrow 0$ with $G$ in $\mathrm{CE}(\mathscr{A})$ and $K$ in $\mathrm{CE}(\mathscr{B})$. Note that $0 \rightarrow \mathrm{Z}_{i}(K) \rightarrow \mathrm{Z}_{i}(G) \stackrel{\widetilde{f}_{i}}{\longrightarrow} \mathrm{Z}_{i}(C) \rightarrow$ 0 is exact since $\mathrm{Z}_{i}(K)=\widetilde{E}_{i}$ and $\mathrm{Z}_{i}(G)=\widetilde{D}_{i}$ by construction. Thus we get that $0 \rightarrow K \rightarrow G \stackrel{\phi}{\longrightarrow} C \rightarrow 0$ is C-E exact by Remark 1.6. Similarly, using the dual result of Lemma 3.8 we can prove that there is a $\mathrm{C}$-E exact sequence 
$0 \rightarrow C \rightarrow H \rightarrow G \rightarrow 0$ with $H$ in $\mathrm{CE}(\mathscr{B})$ and $G$ in $\mathrm{CE}(\mathscr{A})$. This completes the proof.

Let $R$ be a right coherent ring, and let $\mathscr{G} \mathscr{C}=\left\{M \in R\right.$-Mod $\mid \operatorname{Ext}_{R}^{1}(G, M)=$ 0 for any Gorenstein flat $R$-module $G$ \}. Then, by [8, Theorem 2.11], $(R$-Gorflat, $\mathscr{G} \mathscr{C})$ is a complete cotorsion pair. Now the next result follows by [4, Theorem 9.4] and Theorem 3.9.

COROLlary 3.10. If $R$ is a right coherent ring then ( $\mathrm{CE}(R$-Gorflat), $\mathrm{CE}(\mathscr{G} \mathscr{C}))$ is a hereditary and complete cotorsion pair in $C(R$-Mod) relative to $\overline{\operatorname{Ext}}^{1}(-,-)$.

COROLLARY 3.11. If $R$ is a right coherent ring then any complex has a C-E Gorenstein flat cover.

Proof. By Corollary 3.10, the cotorsion pair (CE $(R$-Gorflat), $\mathrm{CE}(\mathscr{G} \mathscr{C}))$ relative to $\overline{\mathrm{Ext}}^{1}(-,-)$ is complete. Then it is easily seen that any complex $C$ has a C-E Gorenstein flat precover and so it has a C-E Gorenstein flat cover by [15, Proposition 1] since the class of C-E Gorenstein flat complexes is closed under direct limits.

\section{REFERENCES}

1. Akinci, K. D., and Alizade, R., Special precovers in cotorsion theories, Proc. Edinb. Math. Soc. 45 (2002), 411-420.

2. Bennis, D., Rings over which the class of Gorenstein flat modules is closed under extensions, Comm. Algebra 37 (2009), 855-868.

3. Ding, N. Q., and Chen, J. L., Coherent rings with finite self-FP-injective dimension, Comm. Algebra 24 (1996), 2963-2980.

4. Enochs, E. E., Cartan-Eilenberg complexes and resolutions, J. Algebra 342 (2011), 16-39.

5. Enochs, E. E., Estrada, S., and García Rozas, J. R., Locally projective monoidal model structure for complexes of quasi-coherent sheaves on $P^{1}(k)$, J. Lond. Math. Soc. 77 (2008), 253-269.

6. Enochs, E. E., and García Rozas, J. R., Tensor products of complexes, Math. J. Okayama Univ. 39 (1997), 17-39.

7. Enochs, E. E., and Jenda, O. M. G., Relative homological algebra, De Gruyter Expos. Math. 30, De Gruyter, Berlin 2000.

8. Enochs, E. E., Jenda, O. M. G., and López-Ramos, J. A., The existence of Gorenstein flat covers, Math. Scand. 94 (2004), 46-62.

9. Enochs, E. E., Jenda, O. M. G., and Torrecillas, B., Gorenstein flat modules, J. Nanjing Univ. Math. Biquarterly 10 (1993), 1-9.

10. García Rozas, J. R., Covers and envelopes in the category of complexes of modules, Res. Notes Math. 407, Chapman and Hall/CRC, Boca-Raton 1999.

11. Gillespie, J., The flat model structure on Ch (R), Trans. Amer. Math. Soc. 356 (2004), 33693390.

12. Göbel, R., and Trlifaj, J., Approximations and endmorphism algebras of modules, De Gruyter Expos. Math. 41, De Gruyter, Berlin 2006. 
13. Holm, H., Gorenstein homological dimensions, J. Pure Appl. Algebra 189 (2004), 167-193.

14. Holm, H.,Gorenstein derived functors, Proc. Amer. Math. Soc. 132 (2004), 1913-1923.

15. Iacob, A., DG-injective covers, \#-injective covers, Comm. Algebra 39 (2011), 1673-1685.

16. Salce, L., Cotorsion theories for abelian groups, pp. 11-32 in: Symp. Math. XXIII, Acad. Press, London 1979.

17. Verdier, J-L., Des catégories dérivées des catégories abéliennes, Astérisque 239 (1996).

18. Yang, G., and Liu, Z. K., Gorenstein flat covers over GF-closed rings, Comm. Algebra 40 (2012), 1632-1640.

DEPARTMENT OF MATHEMATICS

LANZHOU JIAOTONG UNIVERSITY

LANZHOU 730070

P.R. CHINA

E-mail: yanggang@mail.lzjtu.cn

lliangnju@gmail.com 